\title{
Über typische Verätzungen an den Händen der Metallarbeiter, Galvaniseure, Bildhauer etc.
}

\author{
Von
}

\section{Dr. Oskar Neugebauer,}

(Hiezu Taf. I.)

Unter den gewerblichen Hautkrankheiten, denen wir häufig begegnen, können wir zunächst solche finden, bei welchen sich das Individuum bei bestehender Idiosynkrasie nur für sehr kurze Zeit einer Schädlichkeit aussetzt und es infolge des relativ intensiven Reizes rasch zum Ausbruch einer akuten Dermatose kommt. So steht das bei den akut auftretenden Fällen von Ekzem, bei welchen eine kurz dauernde Berührung mit reizenden Substanzen die Erkrankung hervorzurufen im stande ist. Im Gegensatz hiezu gibt es eine Gruppe von Fällen, in welchen die langdauernde und immer wiederkehrende Schädlichkeit die pathologische Veränderung erzeugt, wobei die Schädlichkeit sogar sehr gering sein kann, dafür aber durch die Summierung wirkt. So entsteht, um ein weiteres Beispiel zu nennen, das chronische Ekzem bei den verschiedenen Berufsarten, so entstehen die Bilder, welche sich durch die Einsprengung von Metallteilchen bei Eisen- und Silberarbeitern ergeben, wenn deren Haut immer und immer wieder durch abspringende Splitter (beim Hämmern z. B.) verletzt wird.

Wäbrend diese erwähnten Erkrankungen, vor allem die akuten und chronischen Ekzemformen allgemein bekannt sind 
und auf ihre Entstehung durch die reizenden Agentien schon lange und oft hingewiesen worden ist, sind die Geschwürsbildungen nach Kalkverätzungen, wie wir sie bei Gerbern, Maurern und Galvaniseuren sowie überhaupt bei Metallarbeitern finden, ein noch weniger oft beschrieberies und weniger bekanntes Krankheitsbild. Auch bei diesen Prozessen entsteht durch das schädigende chemische Moment jene Summe von Einzelbildern, die in ihrer Gesamtheit ein für diese Gewerbe in dieser Beziehung gemeinschaftliches und charakteristisches Krankheitsbild liefert. Weil also diesen Verätzungen, beziehungsweise den sich aus ihnen weiter entwickelnden Veränderungen trotz der Verschiedenheit der genannten Berufe einbeitliche Momente zukommen, sollen sie im folgenden besprochen werden.

Was die Literatur über die Fälle gewerblicher Kalkverätzungen der Haut anbelangt, so sei zunächst auf einen Fall hingewiesen, den Löwen $b$ ach (1) in der Wiener Gesellschaft der Ärzte 1903 demonstrierte, mit einer Hautaffektion, die er als Berufskrankheit der Gerber bezeichnete. Der Kranke zeigte "am Mittelfinger der linken Hand einen wie mit einem Locheisen ausgeschlagenen, scharf begrenzten, bohnengroßen Substanzverlust mit glatter, hellroter, glänzender Basis und leicht speckigem Belag von ovalem Kontur obne Reaktionserscheinungen in der Umgebung". Einige kleinere, gleiche Substanzverluste waren an den übrigen Fingern der Hände zu sehen. Solehe Geschwürsbildungen, sagte Löwenbach in seinen Ausführungen zu dem vorgestellten Falle, kämen auch bei Maurern, Gips- und Kalkarbeitern, sowie Stukkateuren vor. Von deutschen Autoren sei uur in Schlegels Übersetzung des Ramazzinischen Werkes 1778 das Vorkommen von Geschwüren bei Gerbern erwähnt, während von französischen Autoren die Affektion des Öftern beschrieben wurde. Bei unseren Arbeitern sei die Erkrankung lange bekannt und werde als Stieglitz bezeichnet, sie scheine aber viel seltener zu sein als in Frankreich. Löwenbach hielt die Affektion für den Effekt der Ätzung durch Kalkpartikelehen, welche diese Wirkung um so intensiver entfalten, als die Haut bei diesen Arbeitern sich dureh die dauernde Wassereinwirkung in einem stetigen Mazerationszustande befinde. Auch Ehrmann (1) gab an, daß die mit gelöschtem Kalk Arbeitenden gerade diese Form zeigen, während bei Arbeitern, die mit Mörtel zu tun haben, Ekzeme entstehen. Die umschriebene Form erklärt Ehrmann damit, daß die gelockerte Epidermis abgerieben wird und dann der ätzende Körper direkt auf die freiliegende Leberhant einwirkt.

Dr. Levy Sirugue (2) behandelt in dem Artikel „Les Dermatoses professionelles" unter dem Abschnitt Dermatoses professionelles à formes definies nebst der Chlorakne, den Papillomen bei Kreosot und Petroleumarbeitern und andern Krankbeitsbildern auch diese "durch ihren speziellen 
Charakter sehr interessante Gewerbedermatose, bekannt unter dem Namen "le pigeonneau", die vor allem durch Lhuillier (3) einem eingehenden Studium unterzogen wurde. Man sieht diese Krankheit besonders bei Lederarbeitern, dann aber auch bei den Polierern der Neusilberwerkstätten, bei den Bleichern, Gipsern und Maurern. In dem Stadium der vollen Ausbildung ist die Läsion eine kleine, kraterförmige Exulceration. Am Rande sieht man einen glatten glänzenden und barten Wulst. Die Öffnung ist wie mit einem Meißel ausgeschnitten; die Tiefe beträgt $1-5 \mathrm{~mm}$, der Durchmesser 2-10 $\mathrm{mm}^{4}$. Bezüglich der Nomenklatur glaubt B r o c q (2) den sonderbaren Namen auf die Ähnlichkeit mit einem Vogelauge zurückführen zu können, während andere Autoren der Ansicht sind, daß die Lebhaftigkeit der Schmerzen die Erkiankten singen ließe und daß hiedurch die Vogelnamen: le perdrix, le rossignol, Je pigeonneau ihre Erklärung fänden. Merkwürdigerweise hat, wie schon oben erwähnt, die Erkrankung auch bei unseren deutschen Arbeitern einen Vogelnamen.

In der Arbeit von B r o c q u. L a u b r y (2a) : „Le pigeonneau. Ulcérations professionelles en pean" ist die in Rede stehende Erkrankung in ausführlicher Weise behandelt und es sind dieser Arbeit auch die Photographien mehrerer Fälle beigegeben. Die Autoren zitieren als erste Arbeit in dieser Frage die von Armieux aus dem Jahre 1853, der bereits neben der "choléra des doigts" auch eine zweite Krankheit der Weißgerber „le rossignol" erwähnt. Diese führt auch Beaugrand (1862) an als eine eigentümliche Affektion der Finger, die nicht mit dem Panaritium verwechselt werden darf. Er sagt: „Die Affektion zeigt sich in der Form einer kleinen Öffnung, von der man glauben möchte, sie sei mit dem Meißel ausgeschlagen. Der Rand ist weißlich und dieser weiße Rand seinerseits ist wieder von einem mehr oder weniger roten Hof umgeben. Das Ganze geht nicht über den Durchmesser einer Linse." - Nach Anführung und teilweiser. Wiedergabe einiger weiterer Autoren (Bazin, La yet etc.), die alle als Ursache der von ihnen beschriebenen Affektion das Hantieren mit Kalk und Auripigment angeben, Substanzen, welche die Arbeiter mehr weniger benützen, beschreiben Brocq und Laubry 3 Fälle ihrer eigenen Beobachtung ${ }^{1}$ ) und fassen dann die sich aus den Krankheitsbildern ergebenden Symptome in folgender allgemeiner Beschreibung zusammen: Im ausgebildeten Zustand besteht "le pigeonneau" in einer mehr weniger beträchtlichen Ulzeration, gewöhnlich von $3 \mathrm{~mm}$ bis $1 \mathrm{~cm}$ lang, 2-6 $\mathrm{mm}$ breit. Sie ist fast immer oval, mit der großen Achse gewöhnlich parallel der großen Achse der Finger, mitunter aber auch senkrecht auf diese. Der Rand der Ulzeration ist oft gebildet durch eine Art Wall von 2-3 mm Dicke, rot oder rosa gefärbt, häufig nach außen wieder durch eine feine Epidermisabhebung begrenzt. Die Ulzeration selbst ist sehr charakteristisch, sie ist steil ausgeschnitten, 2-4 $\mathrm{mm}$ tief, der Grund ziemlich unregelmäßig, je nach dem Stadium der Entwicklung. Oft, wenn die Kranken zur Ordination kommen, ist die Ulzeration vollständig bedeckt mit einer

1) Hiezu mehrere Abbildungen. 
schwärzlichen harten Kruste, die fest haftet und in dem erwähnten Wulst tief eingebettet ist, so zwar, daß die Läsion Äbnlichkeit mit einem Vogelauge hat, was vielleicht der Ursprung der Namen gewesen ist, die die Arbeiter der Affektion gegeben haben. Nach einigen anderen Autoren aber sind es die heftigen Schmerzen, welche die Kranken singen lassen, woher der Name "rossignol“ kommt.

Bezüglich der Entstehung äußern sich die Autoren dahin, daß, so lange die Epidermis intakt ist, keine Erkrankung eintritt, die kaustischen Substanzen aber sofort ihre Wirkang langsam und kontinuierlich ausübeu, sobald sich irgendwo an den Fingern eine Läsion der Oberhaut etabliert hat. Diese Pathogenie erklärt also ganz natürlich: 1. die Entstehung durch eine Fissur, Erosion etc., 2. die Weiterentwicklung der Läsion, 3. das charakteristische Aussehen der Wunde, die steil ausgeschnittenen Ränder, den peripheren Entzündungswall etc., 4. warum der Anblick den Arsenulzerationen der Hände so sehr gleicht. Es handelt sich bei all' diesen Läsionen um eine langsame kaustische Zerstörung der durch was immer für eine Ursache ihrer Hornschicht entblößten Epidermis.

Den Grund zur Etablierung der Geschwüre sehen die Autoren entgegen ibrer ursprünglichen Ansicht nicht in der Einwirkung des Auripigments oder Kalks, vielmehr in der direkten Einwirkung der Flüssigkeiten, besonders der Beizen. ${ }^{\text {) }}$ )

Im Handbuche der Arbeiterkrankheiten sagt Dreyfuß (4) im Abschnitt über die Krankheiten der Gerber: „Besonders erwähnt sei, weil nur bei Gerbern bescbrieben, die sogenannte Täubchen- oder Nachtigallengeschwulst (le pigeon, pigeonneau, rossignol). Es ist dies nach Layet (5) ein kleiner wie mit einem Locheisen ausgeschlagener Substanzverlust. Die Ränder sind weißlich und dieser Rand ist wieder mit einem mehr oder weniger dunkien Hof umgeben. Die kleine Effloreszenz ist je nach der Individualität mehr oder weniger schmerzhaft. " - In Bezug auf die Ätiologie gibt Dreyfuß für die Frkrankung an, daß sie als besonders häufigé Folge des Koutaktes mit Ätzmitteln (Kalk) auftrete.

An dieser Stelle möge einiges über die einschlägige Beschäftigung der Gerber gesagt werden, damit dadurch verständlich wird, wieso die Gerber zu diesen Kalkverätzungen kommen. - Damit die Lederbaut (das Leder) von der Epidermis und den Haaren getrennt wird, ist es notwendig, entweder einen leichten Fäulnisprozeß der Häute hervorzurufen, oder diese der Einwirkung ätzender Substanzen zu unterziehen. Als Ätzmittel wird $\mathrm{Ca}(\mathrm{OH})_{2}$ angewendet, und $\mathrm{zwar}$ kommen die Häute in Behälter, in denen zunächst Ätzkalk ( $\mathrm{CaO}$ ) gelöscht wird, worauf dann die Felle in die erkaltete Flüssigkeit eingelegt werden. Nachdem diese genügend lange Zeit eingewirkt hat, werden sie mit großen Zangen herausgenommen

1) In diesem einen Punkte dürften unsere Beobachtungen zu einem anderen Resultate führen, denn 1. haben einige Kranke nur mit Kalk zu tun gehabt, 2. aber baben wir gerade in den Krusten noch Kallsteilchen gefunden. (Näheres später.) 
und müssen dann durch Handarbeit von den Haaren und der Oberhaut befreit werden, ebenso wie auch das noch zum Teil haftende Unterhautzellgewebe und die gleichfalls noch vorhandenen Reste der Muskulatur nach neuerlichem Einlegen in schwächere Kalkmischungen entfernt werden müssen. $\mathrm{Da} B$ es bei diesen Manipulationen vorkommt, daß an den Häuten befindliche Kalkpartikelchen in die Finger eingerieben werden, ist leicht verständlich, ebenso $\mathrm{daB}$ diese auch dort verbleiben, wenn nicht gründliches Waschen erfolgt. Außerdem ist auch zu bedenken, daß die Arbeiter bei ihrer Tätigkeit gewiB nicht zu sorgsam in Bezug auf ihre eigene Person sein können und daß sie sich hiedurch leicht Verletzungen an den Händen zuziehen, aus welchen der eingedrungene Kalk um so schwerer zu entfernen ist. $\left.{ }^{1}\right)$

Was des Weiteren einschlägige Krankheitsbilder anlangt, so sei ein Fall erwähnt, den Groß (6) in der Wiener dermatologischen Gesellschaft vorstellte, bei welchem bei einem Arbeiter, der beim Abladen von Rohsoda beschäftigt ist, Nekrosen der Haut an Händen und Unterarmen in verschiedener Größe auftraten. Der zentrale Schorf ist scharf abgegrenzt, trocken, fällt spontan ab und hinterläßt einen scharf begrenzten, gewöhnlich kreisrunden Substanzverlust, der alle Schichten der Haut betrifft. Das Präparat (die Soda) ist sehr wasserarm, zieht, auf die Hant gebracht, aus den Geweben gierig Wasser an, damit ist auch die Entstehung eines völlig trockenen Schorfes geklärt.

Auch E hrmann(6) hat vor mehreren Jahren in der Gesellschaft der Ärzte eine Reihe von solchen Fällen gezeigt, bei welchen punkt- und linsenförmige Schorfe bestanden. Die Fälle waren lange Zeit unerklärlich, bis sich schließlich ergab, daß die Leute an der Donau aus Bosnien kommende Soda abluden. Die bei einer kleinen Menge von Doz. R i e $B$ angestellte Untersuchung ergab, daß anch hier keine Kristalle von Natronlauge sondern kalzinierte Soda vorlag. Ehrmana selbst gelang es nicht an sich selbst Erscheinungen zu erzeugen.

Áhnliche Fälle von Sodaverätzungen gesehen zu haben gibt auch Zumbusch (6) an, während Riehl (6) auf Fälle hinweist, die er in einer Bürgerschule in Leipzig beobachten konnte, in welchen sich die Mädchen die Haut mit Kochsalzkristallen verletzten und Gangrän auftrat, trotzdem Salz kein Ätzmittel ist. Zum Schluß sei noch auf den vor kurzem in der Wiener dermatologischen Gesellschaft vorgestellten Fall hingewiesen, den Kirsch (6) voratellte: Am linken Mittelfinger der Patientin fand sich an der medialen Fläche ein etwa kleinfingernagelgroßer, scharfrandiger, ovaler bis in die Subcutis reichender Substanzverlust. Die Patientin gab an, mit der Reinigung von Nickel- und Silber-

1) Hier sei auch erwähnt, daß mir angegeben wurde, dab die Gerber, um ibre Hände dem Eindringen der Kalkteilchen gegenüber möglichst widerstandsfähig zu machen, tägliche Handbäder in konzentrierter Lohbrühe gebrauchen undihre Hände dann im Rauche angezündeten feuchten, Heues trocknen. 
ketten beschäftigt zu sein, wobei sie die Ketten stramm um die 3 letzten Finger der linken Hand geschlungen hat und sie gegen eine von einem Gemisch von Seifenwurzeldecoct und Schwefelsäure berieselte Bürsten. walze hält. Beim Zustandekommen der dem Geschwür zu Grunde liegenden Hautnekrose soll nicht nur eine chemische, sondern auch eine mechanische Noxe, nämlich die Kompression der Gewebsteile durch die straff gespannte Kette eine Rolle spielen.

Was unsere Fälle anlangt, so seien hier die diesbezüglichen Daten von zehn Fällen angegeben, die wir im Verlaufe von ungefähr zehn Monaten in der Ambulanz des Dozenten M. 0 p p e n heim zu verzeichnen hatten.

1. T., Hilfsarbeiterin in einer Metallfabrik: An der Innenseite der Finger beider Hände finden sich ovale, daneben auch kreisrunde, immer aber scharf begrenzte Substanzverluste von höchstens Linsengröße; dieselben erscheinen wie mit einem Locheisen ausgeschlagen. Die Basis dieser Substanzver]uste ist glatt und schmerzhaft. Die Ulzerationen sind zum Teil von einem nur wenig geröteten Wall, der sich etwas hart anfühlt, umgeben, einige andere hingegen haben auch diesen Rand nicht, sondern grenzen makroskopisch an normale Haut.

2. K., Hilfsarbeiterin. An den Fingern finden sich an mehreren Stellen Kalkverätzungen in Form von scharf begrenzten Geschwüren mit nur geringen Reaktionserscheinungen in der Umgebung.

3. C., Hilfsarbeiterin, deren Beschäftigung in dem Waschen von Geschirren mit Kalk besteht; auch bei ihr finden sich mehrfach an den Endphalangen der Finger kreisrunde, mit braungelben, glatten und festhaftenden Krusten bedeckte Effloreszenzen von etwa Hanfkorngröße, die nur von einem leicht roten Hof eingesäumt sind.

4. K., Hilfsarbeiterin in der Metallfabrik C. (Beschäftigung s. u.) Die rechte Hand der Patientin zeigt runde Substanzverluste von scharfer Umgrenzung, so daß sie wie ausgeschnitten erscheinen. Befallen sind der Handrücken und die Gegend dorsal von den Interdigitalfalten. Die Patientin bietet ein vollkommen analoges Bild mit der gleichzeitig erscheinenden

5. L., die Hilfsarbeiterin in derselben Fabrik mit derselben Beschäftigung wie $K$. ist.

6. B., Hilfsarbeiterin in einer Metallwarenfabrik, wo sie mit Kalk und Vitriol zu arbeiten hat. Es finden sich bei ihr an den Innenseiten der Finger ovale bis kreisrunde, ziemlich tiefe Substanzverluste von Hanfkorn- bis unter Erbsengröße von bedeutender Schmerzhaftigkeit Diese Substanzverluste sind von einem derben geröteten Wall umgeben.

7. M., Hilfsarbeiterin gleich 4-5; die Patientin steht in unserer Ambulanz wegen Ekzema manus in Behandlung, das durch Hantieren in den Nickelbädern verursacht sein soll. Patientin hat durch längere Zeit eine Arbeiterin bei der "Kalkarbeit" vertreten müssen. Sie zeigt neben dem Ekzem, das sich über beide Hände erstreckt, namentlich an den 
Gelenksbeugen der Finger zum Teile scharf umschriebene Herde, die mit gelhbraunen Krusten bedeckt sind. An der Kuppe des kleinen Fingers sieht man ein Kalkkörnchen in die Kruste eingesprengt, das entfernt wird. An anderen Stellen sind die Krusten bereits abgelöst und unter ihnen tritt ein im Vergleich zur Oberflächen. ausdehnung tiefer, scharf umschriebener Substanzverlust von glatter Basia zu Tage. Patientin macht über diesen Teil ihrer Erkrankung folgende Angabe: Zuerst bemerkt sie die Kalkkörnchen, dann bildet sich um diese herum eine Kruste, die zunächst festhaftet, dann oft abgerissen wird, worauf dann „ein Loch" in der Haut sei.

8. S., Bildhauer. ${ }^{1}$ ) An den Fingerspitzen einige nicht sehr tiefgreifende Ulzerationen ohne besondere Reizerscheinungen in der Umgebung. Die Geschwüre sind etwa hanfkorngroß und ungefähr kreisrund bis oval, wobei aber die Konturen nicht besonders scharf gezeichnet erscheinen, sondern vielmehr etwas gezackt sind. Die Basis der Substanzverluste ist dunkler rot als die der vorhergehend erwähnten Kranken. An der Kappe des linken Daumens ist ebenfalls ein etwa kleinfingernagelgroßes Gebiet erkrankt, hier zeigt sich aber keine Ulzeration, sondern die Haut ist von Rissen durchsetzt und macht mehr den Eindruck eines rhagadiformen Ekzems. Die Affektion ist ziemlich schmerzhaft und behindert den Patienten so sehr, daB er nur schwer in der Lage ist, Gegenstände mit den Fingern aufzunehmen.

9. S., Hilfsarbeiterin in einer Metallwarenfabrik, aus der uns auch andere Fälle von gleichen Verätzungen zugegangen sind. Die Patientin zeigt an beiden Händen multiple Verätzungen, und zwar zum Teil bereits freiliegende Substanzverluste, zum Teil solche Substanzverluste der Haut, die noch mit einer Kruste bedeckt sind. Die Verätzungen finden sich vorwiegend zwischen den Fingern sowie an den Interphalangealgelenken, nur wenige am Dorsum manus, Sie sind etwa hanfkorngroß, was die größere Zahl von ihnen anbelangt, einige etwas kleiner. Ibre Form ist kreisrund, auch oval, auffallend ist der scharfe Rand. An mehreren Stellen sieht man in die Krusten kleine Kalkpartikelchen eingesprengt, die nur schwer aus ihren Vertiefungen zu entfernen sind. Die Schmerzhaftigkeit der Ulzerationen, in deren Um. gebung Reaktionserscheinungen fehlen, ist ziemlich hochgradig.

10. W., Hilfsarbeiterin in einem galvanischen Betriebe. An der Innenseite ${ }^{2}$ ) des Interphalangealgelenkes I/II des rechten 4. Fingers etwas dorsal ein etwa bohnengroßer ovaler Substanzverlust der Haut mit scharfen Konturen, bedeckt mit einer gelbbraunen Kruste, die fest haftet. Die Umgebung des Substanzverlustes ist leicht gerötet. Ein etwas kleinerer,

1) Ein Arbeiter des Patienten, der mir gestattete in sein Atelier zu kommen, bot ein dem Erkrankten ähnliches Bild, da auch er am Daumen einen Substanzverlust aufwies, den er auf das Hantieren mit Kalk (s. u.) zurückführte.

2) Siehe Abbildung. 
ebenso beschaffener Herd an dem Interphalangealgelenk II/III des"linken Mittelfingers, Außerdem mehrere scharf ausgeschnittene Substanzverluste, kreisrund bis oval, mit glatter Basis ohne Reaktionserscheinungen in der Umgebung, zum Teil noch mit einer festhaftenden Kruste bedeckt oder - wenn dies nicht mehr der Fall ist - tiefgreifend, an den Fingern beider Hände. - An der Innenseite des rechten 4. Fingers anßerdem eine ovale, scharf umschriebene glatte Narbe ron etwa Bohnengrölie, etwas unter dem Niveau der Haut. Mehrere solche am Mittelfinger der linken Hand, am linken 2. und 4. Finger sowie am Handrücken. Diese Narben rühren nach Angabe der Patientin von Verätzungen her, welche sich diese im Jahre 1892 oder 1894 bei der Kalkarbeit in der Fabrik B. zugezogen hatte.

Die erkrankten Stellen sind sehr schmerzhaft; die Patientin hält dementsprechend die F'inger leicht gebengt und steif; sie ist im freien Gebrauch ihrer Hände ziemlich eingeschränkt. Bei dieser Patientin soll auch aus dem weiteren Verlaufe berichtet werden. Nachdem diese nämlich durch einige Tage sich zu Hause gepflegt hatte, waren die Ulzerationen unter Borsalbenverband (den wir in diesen Fällen gewöhnlich auch sonst anwendeten) so weit. gebessert, $\mathrm{da} B$ sie entgegen unserm Rate, ihrem Berufe wieder nachging. Nach ungefähr 10 Tagen stellte sich Patientin wieder in unserer Ambulanz vor, da sich ihre Krankheit so weit verschlechtert hatte, daß ihr das Arbeiten unmöglich wurde. Sie bot jetzt neben den Verätzungen, die im vorhergehenden beschrieben wurden und die noch deutlich sichtbar waren, nachstehende neue Erscheinungen. (Die Abbildung 3 und 4 repräsentieren den Zustand der Patientin während des ersten Krankenstandes, daher fehlen in ihr die im folgenden beschriebenen Veränderungen.)

Am Dorsum der Grundphalanse des linken Mittelfingers zwei stecknadelkopfgroße, kreisrunde Krusten von gelbbrauner Farbe mit randständigen Kalkpartikelchen. Diese Krusten sind nach einem Tage unter Borsalbenverband abgefallen und unter ihnen zeigen sich wieder zwei entsprechend große, kreisrunde Substanzverluste mit hellrotem, glatten Grund. Am Interphalangealgelenk II/III des linken kleinen Fingers wieder eine oval konturierte Kruste von etwas weniger als Bohnengröbe, im Zentrum braun, gegen den Rand zu mehr gelb, ohne Rötung der Umgebung, eine ebensolche am Interphalangealgelenk I/II des linken 4. Fingers. Interdigital, jedoch noch am 4. Finger zwei kleinere ähnliche Krusten, örtlich von einander getrennt, jedoch von einem gemeinschaftlichen roten Hof umgeben. Volar am rechten Daumen, nahe der Kuppe ein etwa hanfkorngroßer, schwärzlich belegter Substanzverlust, der sehr schmerzhaft ist. - Das Relief der $\mathrm{Haut}$, welehes durch die Furchen gezeichnet wird, erscheint besonders ander ulnaren Seitedes linken Dors um manus und in der linken Palma bis in die feinsten Verästelungen weiblich nachgezeichnet. Diese Zeichnung rüht von den hier abgelagerten feinen Kalkteilchen her und kann selbst durch 
gr ündliches Waschen von ihr nichtentfernt werden. Etwas weniger deutlich erscheint die Zeichnung an Thenar der rechten Hand. ) Abgesehen von diesen in den vorhergehenden Absätzen geschilderten streng lokalisierten Veränderungen ist die Haut der Hände im allgemeinen leicht von Rhagaden durchsetzt und etwas gerötet; sie bietet stellenweise das Bild eines entstehenden Ekzems, das aber hinter den eigent. lichen Verätzungen zurücktritt.

Überblicken wir demnach die hier verzeichneten Fälle, so sehen wir, abgesehen von Fall 8, folgendes Bild: An den Händen, öfters an den Innen- und Beugeflächen der Finger, ebenso auch an den Gelenken und zwischen den Fingern finden sich in größerer Zahl Krusten von etwa Hanfkorn- bis über Linsen- oder Bobnengröße, von gelbbrauner Farbe, gewöhnlich kreisrund bis oval, dabei aber immer scharf umschrieben; um sie herum entweder normale Haut oder eine nur leichte Rötung, beziehungsweise ein nur etwas geröteter Wall. Nach Ablösung dieser Krusten, sich aber zumeist als schon vorhanden präsentierend und die Zahl der Krusten überhaupt übertreffend, zeigen sich uns Ulzerationen, die kreisrunde bis ovale, fast nie unregelmäßige Gestalt besitzen und daher wie ausgeschnitten erscheinen; diese Ulzerationen haben höchstens einen Durchmesser von ca. $1 \mathrm{~cm}$, gewöhnlich sind sie aber viel kleiner, auffallend aber ist ihre im Vergleich zum Durchmesser bedeutende Tiefe. In der Umgebung dieser Substanzverluste sind die Reaktionserscheinungen, wenn überhaupt vorhanden, gering, es besteht gewöhnlich nur ein leicht roter, ziemlich schmaler Hof, der sich mitunter auf einem die Ulzeration nach außen umgebenden leicliten Wall vorfindet. Die Ulzerationen selbst sind gewöhnlich ziemlich schmerzlaft und beeinträchtigen die Patienten bei ihrer Arbeit. Ferner zeigten einige Fälle im Zentrum der Geschwüre weiße, schwer entfernbare Kalkpartikelchen, was den Entstehungsmodus mancher dieser Geschwüre erklärt. Im Falle 8 hingegen, selbstverständlich ohne aus diesem einzelnen Falle einen Schluß ziehen zu wollen, bestand ein etwas abweichendes Bild

1) Patientin gibt an, daß3 in den Betrieben zur Reinigung der Hände nach der Kalkarbeit Salpetersäuremischung verwendet wird, welche den Kalk auföst. An ihrer gegenwärtigen Arbeitsstätte stand der Patientin nach ihrer Angabe diese Art der Reinigung nicht zu Gebot. 
insoferne, als die Ulzerationen nicht so scharfe Konturen zeigten und von geringerer Tiefe waren und daß an einer erkrankten Stelle sich eher ein Ekzem als eine Geschwürsbildung zeigte.

In den Fällen $3,4,5,6,7,8,9$ und 10 sind auch Angaben über die Tätigkeit der Erkrankten angegeben und zwar haben insbesondere die Patientin beziehungsweise Patientinnen 7 (mit der gleichen Beschäftigung wie 4, 5 und 9), dann 8 und 10 genauere Daten bezüglich der von ihnen zu verrichtenden Arbeit gegeben, die hier verzeichnet werden mögen :

Patientin 7 arbeitet in einer Metallfabrik, in welcher Metallgegenstände vernickelt werden. Zunächst werden diese mit Petroleum gewaschen, dann mit gelöschtem Kalk behandelt, hierauf mit reinem Wasser von den Kalkteilchen wieder abgewaschen, worauf sie dann in die Nickelbäder kommen.

Patientin 10 gibt folgendes an: Gegenstände, die vergoldet oder vernickelt werden sollen, müssen vorher mit $\mathrm{Kalk}$ behandelt werden. Sie kommen zunächst vom Polierer, wo sie eingefettet worden sind. Jetzt müissen sie je nach der Beschaffenheit mit Bürsten oder Tüchern, die mit Kalkmilch getränkt sind, abgerieben, sodann mit reinem Wasser abgewaschen werden; dann kommen sie in die Nickelbäder, wo sie an Drähten aufgehängt sind. Patientin, die mehrere dieser Arbeiteń zur selben Zeit oder wenigstens bald nacheinander verrichten mußte, hat, um rasch arbeiten zu können, den Draht jedesmal nicht abgeschnitten, sondern abgerissen und sich. da er dünn war, Schnittwunden an den Fingern zugezogen. Da sie bald wieder die "Kalkarbeit" verrichten mußte, konnte jetzt die ätzende Wirkung leicht eintreten, da die Patientin sich vorher Verletzungen ihrer Haut ausgesetzt hatte. - Die Patientin hatte wäbrend ihrer Arbeit keine Schutzhandschuhe an, die vorerwähnten Patientinnen hingegen nur Fingerlinge, sagen aber, daß auch diese nur einen unvollständigen Schutz gegen das Hineinfließen der Flüssigkeit gewähren.

Verschieden pon diesen beiden, einander sehr äbnlichen Angaben sind diejenigen, welche uns Patient 8 machte: Zunächst arbeitet er nur wenig mit Weißkalk $\mathrm{Ca}(\mathrm{OH})_{2}$, sondern zumeist mit Zement und Portlandkalk. Patient macht aus diesem Kalk und Wasser einen Brei, der rasch verarbeitet werden muB, 
weil er an der Luft schnell trocken und hart wird. Außerdem benötigt der Patient zu seiner Arbeit ein Gemenge von Sand und Wasser, so daß die Arbeiten erstens gröBtenteils mit nassen Händen verrichtet werden müssen und zweitens auch durch die kleinen kantigen und spitzigen Steinchen in der obgenannten Mischung zu leichten Hautverletzungen an Fingern und Händen führen, besonders dann, wenn die Leute noch nicht das Hantieren mit dem Sand gewohnt sind und eine feinere Haut besitzen. So war es auch bei dem Patienten, der einen seiner erkrankten Gehilfen vertreten mußte und sich der ihm nicht mehr gewohnten Beschäftigung unterziehen mußte. Nach kurzer Zeit konnte er diese nicht mehr ausführen und kam in unsere Ambulanz. Bemerkt sei noch, daß sowohl er als seine Gehilfen besonderes Gewicht darauf legten, da $\beta$ die Haut konstant naß gehalten werden müsse, damit die Verätzungen entstünden und daß mir gesagt wurde, daß diese besonders bei Neulingen im Berufe aufträten. (Der Patient, ein intelligenter Mann, bezeichnete die Affektion als „Kinderkrankheit, die jeder mitmachen müsse ${ }^{4}$.)

Um die Wirkung des Kalkes auf die Haut experimentell zu untersuchen, habe ich einige Versuche angestellt, wobei ich mir aber gleich von Anbeginn an klar war, daß das Resultat, wenn überhaupt nur ein partielles sein konnte. Denn ich glaube, daß abgesehen von der Einwirkung des Kalkes, die in Frage stehenden Ulzerationen wohl noch durch die Mitwirkung anderer Falstoren erzeugt werden, die gewiß noch eine unterstützende Rolle spielen, Säuren, sich erst neu bildende chemische Verbindungen, die alle in Gemeinschaft mit dem Kalk auf die Haut einwirken, so daß diese einer ganzen Summe ron chemischen Agentien ausgesetzt ist.

Von einem etwas andern Standpunkte aus aber - glaube ich - ist zu bedenken, daß ein großer Unterschied besteht, ob man experimentell ein Kalkkörnchen einreibt und dann die Stelle in Ruhe läßt, oder ob der Arbeiter sich bei der Arbeit Kalk in Verletzungen einreibt, auf diese aber nicht achtet, bis er durch die Schmerzen gezwungen wird, nachzusehen. Was mag sich bis dahin ereignet haben, was wir im Experiment nicht ausführen können? Immerhin aber muß, wenn der Kalk 
bei diesen Krankheitsformen eine wichtige oder vielmehr die wichtigste Rolle spielt, das Experiment ein äbuliches Resultat ergeben, als uns die Erkrankten, die sich durch ibre berufliche Tätigkeit ihre Krankheit zugezogen haben, in dieser darbieten. Betont ist von den Schädlichkeiten, die hier mitwirken, besonders eine worden, nämlich das Wasser und daher ist auch diesbezüglich ein Versuch angestellt worden, über den berichtet werden wird. Nach diesen einleitenden Bemerkungen, die dartun wollen, daß ich mir bewußt bin, daß der Kalk bei den vorliegenden Erkrankungen nur eines der ursächlichen Momente, voraussichtlich ja das wichtigste ist, wenn es nur von den anderen Hilfsursachen begleitet wird, will ich diese Versuche anführen, die ich an mir selbst angestellt habe, um die Wirkung des Kalkes auf die menschliche Haut zu untersuchen.

Experiment I. Einreibung von $\mathrm{Ca}^{\prime}(\mathrm{OH})_{2}$ in intakter Hant.

14./IV. Von dem bei Patienten 8 in Verwendung stehenden Weißkalkbrei wurde ein Quantum auf die Haut des linken Vorderarmes an der Volarseite eingerieben.

15./IV. Der Kalk an die Haut angetrocknet, jedoch fehlt jede Spur einer Reaktion.

16./IV. Derselbe Befund, der Kalk bröckelt sich ab.

Experiment II. a) Einreibung von $\mathrm{Ca}(\mathrm{OH})_{2}$ in die oberfächlich verletzte Haut.

11./IV. Wieder an einer Stelle der Volarseite des linken Vorderarmes wurde an der Ulnarseite die Haut leicht aufgekratzt, so dab an einzelnen Stellen der so entstandenen Hautverletzung leichte Blutung eben auftrat. Die Kratzwunde war fast linear und etwa $3 / 4 \mathrm{~cm}$ lang. Von dem Kalkbrei wurden einige Körnchen in dieselbe eingerieben. Knapp naehher leichtes Brennen und manchmal ein stechender Schmerz.

12./IV. An der angegebenen Stelle eine gelbbraune Kraste etwa $2 \mathrm{~mm}$ breit, $1 \mathrm{~cm}$ lang, in sie eingesprengt besonders am Rande sichtbar kleine Kalkpartikelchen. Die Umgebung leicht gerötet, das Ganze gegen Berüh. rung etwas schmerzhaft, aber nicht mehr spontan.

15./IV. Die Kruste läßt sich nur schwer ablösen, weswegen hievon Abstand genommen wird.

18./IV. Die leichte Rötung in der Umgebung der Kruste besteht fort. Die Kruste läßt sich heute leichter abbeben und unter ihr zeigt sich ein im Verbältnis zur ursprünglich gesetzten Hautverletzung tiefer Substanzverlust mit glattem Rand und leicht belegter Basis.

19./IV. Über dem Substanzverlust neuerdings eine ziemlich fest haftende Kruste.

21./IV. Die Kruste wird wieder entfernt. Der Substanzverlust zeigt ungefähr Rechtecksform an der Oberfläche, erscheint aber bei näherem 
Zusehen aus drei kleinen glatten Hohlkugeln zusammengesetzt; der Rand, der diese miteinander verbindet, wird von einem kleinen Wall gebildet, der nur leicht gerötet ist.

b) Einreibung von Zementkalk in die oberflächlich verletzte Haut.

11./IV. Radial von der unter a) bezeichneten Stelle wurde die Haut mit dem in Verwendung stehenden Sandgemenge (s. o.) aufgerieben, so $\mathrm{da} G$ sie ebenfalls leicht erodiert war, dann wurden auch hier Zementkalkkörnchen eingerieben, die Stelle aber später nicht mehr befeuchtet. (Der Bildhauer sagte mir, der Zementkalk wirke besonders dann, wenn die Leute gleichzeitig in der Nässe arbeiten.)

12./IV. Die Rötung, die gestern auch hier aufgetreten war, verschwunden, die Stelle überhaupt fast wieder normal.

Experiment II etwas modifiziert wiederholt.

14./IV. Ulnar und radial wurde mit einem Skalpell, dessen Spitze abgebrochen war, durch drehende Bewegung eine oberflächliche Erosion von ungefähr Kreisform mit ca. $2 \mathrm{~mm}$ Durchmesser gesetzt und dann wieder radial Zementkalk, ulnar Weißkalk eingerieben.

15./TV. Die gestern erodierten Stellen mit einer Kruste bedeckt, in welcher man noch die Kalkpartikelehen eingesprengt sieht.

18./IV. Die radiale Kruste (Zementkalk) läßt sich leicht ablösen, unter ihr zeigt sich eine ganz seichte Ulzeration von keiner bestimmten Umgrenzung; sie ist ungefähr dreieckig, keine Blutung. Die ulnare Kruste läbt sich nur schwer und teilweise abjösen.

21./IV. Die ulnare Kruste kann heute abgehoben werden; unter ihr tritt ein Substanzverlust zu Tage, der deutlich tiefer ist als der radiale, der überhaupt heute schon fast abgeheilt ist. Dieser Substanzverlust bat ungefähr Kreisform und ist von einem lichtroten Wall umgeben.

Überblicken wir also die beiden Experimente, so zeigt sich zunächst, daß im Experiment I das Resultat ein negatives war. Beim Experiment II hingegen ergab sich in der Tat aus dem Versuche mit gelöschtem Kalk eine kleine Ulzeration, die mit den Ulzerationen, welche wir bei den Patienten beobachten konnten, in einigen Punkten Ähnlichkeit zeigte, und dies um so mehr, wenn man das fast negative Resultat des Versuches mit Zementkalk in Betracht zieht, bei dem trotz vorhandener Erosionen einmal überhaupt keine nennenswerte Veränderung entstand, beim zweiten Male nur ein relativ ganz oberflächlicher Substanzverlust. Bei dem Versuche mit gelöschtem Kalk hingegen, ist beide Male eine verhältnismäßig bedeutendere Ulzeration entstanden, deren Charakter nochmals erwähnt werden soll: nach Abhebung der ursprünglich auffallend fest haftenden Kruste tritt ein Substanzverlust von scharfer Begrenzung (wenig- 
stens im ersten Falle) zu Tage, der ziemlich tief ist und von einem niedrigen leicht roten Wall eingesäumt wird. Die Schmerzhaftigkeit ist nur gering.

Von den bei der Arbeit der Erkrankten in Betracht kommenden Umständen scheint die Mazeration der Haut durch das Wasser eine Rolle zu spielen; deswegen habe ich in einem weiteren Versuche die Haut der Einwirkung beider Faktoren unterzogen und beschreibe

Experiment III. Einreibung von Weißkalk in die oberflächlich verletzte, vorher aber durch Wasser mazerierte Haut.

22./IV. Vormittag: Um den linken Oberarm wird ein sehr nasser Umschlag gemacht, darüber Billrotbatist gegeben und die Haut durch den Verband gut gegen außen abgeschlossen.

23./IV. Vormittag: Die Haut noch feucht; gleicher Verband Nach. mittag wird an der Volarseite der bezeichneten Stelle durch Drehen mit einer Meißelsonde eine oberfächliche Erosion gesetzt. Die Epidermis läßt sich sehr leicht abschaben; Einreibung von Weißkalk, nachher wieder leichter stechender Schmerz neben dem kontinuierlichen, der bis zum Abend anhält.

24./IV. An der bezeichneten Stelle eine bräunliche Kruste mit eingesprengten kleinen Kalkpartikelchen.

27./IV. Die Kruste kann etwas gelockert werden und wird am

28./IV. entfernt. Es erscheint unter ihr ein Substanzverlust der Haut; diese besitzt die Form eines gleichseitigen Dreieckes von ungefähr $3 \mathrm{~mm}$ Seitenlänge, deren Ecken abgerundet sind. Auffallend ist besonders der scharfe Rarid, so daß es den Anschein hat, als ob die Haut an dieser Stelle ausgezwickt oder ausgeschnitten worden wäre, auch deswegen, weil der Grund des Substanzverlustes, der nur etwas gelblich belegt ist und nicht blutet, ganz glatt ist. In der Umgebung der Ulzeration ein sie nach außen abgrenzender, niedriger eben noch bemerkbarer Wall von leichtroter Farbe, durch welchen die scharfe Begrenzung der Ulzeration noch dentlicher hervortritt. Auch diese Ulzeration weist im Vergleich zu der ursprünglichen Erosion eine ziemlich bedentende Tiefe auf, welche allerdings der Wall, der sie umgibt, noch beträchtlicher erscheinen läßt.

Da uns somit auch dieses Experiment ein den Ulzerationen der Erkrankten ähnliches Bild ergeben hat, indem es uns, wie hervorgehoben, ein tiefes, scharf begrenztes Geschwür mit glattem Grunde lieferte, können wir mit großer Wahrscheinlichkeit den Schluß ziehen, daß auch der gelöschte Kalk im stande ist, namentlich dann, wenn schon Verletzungen der Haut bestehen, Ulzerationen dieses bestimmten, uns sich immer wieder von neuem präsentierenden Typus zu erzeugen. Ob 
außerdem noch chemische Agentien oder andere Momente vorhanden sind, die den Kalk in seiner Ätzwirkung unterstützen und welche es sind, ist in diesen Experimenten nicht untersucht worden, jedenfalls aber dürfte ihnen doch nur eine untergeordnete Wirkung zukommen, während der Kalk bei diesen Erkrankungen der genannten Arbeiter wohl die wesentlichste Rolle spielt. Die Mazeration durch das Wasser, in dem und mit dem die Leute ja fortwäbrend arbeiten müssen, macht die Haut gewiß den Verletzungen leichter zugänglich als bei reiner Trockenarbeit möglich wäre, natürlich unter sonst gleichen Bedingungen.

Zum Schlusse soll noch auf die Bedeutung der besprochenen Erkrankungen eingegangen werden, soweit diese nämlich unsere Patienten in der Ausübung des Berufes hinderten. Was unsere 10 Patienten anlangt, so mußten sie sämtlich als arbeitsunfähig erklärt werden. Die Dauer des Krankenstandes sowie ihr Zustand beim Austritt aus demselben ist in folgender Tabelle zusammengestellt.

\begin{tabular}{|c|c|c|c|c|c|}
\hline \multirow[t]{6}{*}{ Jahr 1907} & & zenstand vom & bis & $=$ Tage & Austritt als \\
\hline & $\mathrm{T}$. & 19. Juli & 29. Juli & 11 & gebessert \\
\hline & $\mathrm{K}$. & $25 . \quad "$ & 1. August & 8 & geheilt \\
\hline & c. & 14. Septbr. & 25. Septbr. & 12 & gebessert \\
\hline & $\mathrm{K}$. & 17. & 21. & 5 & n \\
\hline & H. & 17. & 23. & 7 & $"$ \\
\hline \multirow[t]{5}{*}{ Jahr 1908} & S. & 8. Februar & 22. Februar & 15 & $n$ \\
\hline & B. & 5. März & 17. März & 13 & $"$ \\
\hline & M. & 31. & 7. April & 8 & geheilt \\
\hline & S. & 9. April & 13. $"$ & $\check{5}$ & gebessert \\
\hline & W. & 13. & 21. & 9 & ungeheilt \\
\hline
\end{tabular}

Im Durchschnitt waren also unsere Patienten etwa 10 Tage arbeitsunfähig und auch zur Zeit des Austrittes aus dem Krankenstande noch nicht vollständig geheilt, konnten aber schon dem Berufe nachgehen.

Im Anschluß an die geschilderten Fälle, welche Kalkverätzungen betrafen, möchte ich über drei weitere Fälle berichten, die sich in der letzten Zeit und zwar zufällig fast gleichzeitig in unserer Ambulanz eingefunden hatten, bei welchen die ätzende Wirkung durch Schwefelsäure hervor- 
gerufen wurde. Auch hier handelte es sich nicht um zufällige Verletzung, sondern um eine durch den Mechanismus der Arbeit bedingte Gewerbekrankheit. Zunächst sollen die Befunde der Krankheitsbilder verzeichnet werden und dann einige Angaben über die Art der Tätigkeit der Patienten, die zu den im nachstehenden beschriebenen Verätzungen führte.

1. St. An der radialen Seite des Interphalangealgelenkes II/III des rechten 4. Fingers ein über bohnengroßer Substanzverlust, der scharf konturiert ist. Der Rand des Ovals ist aber kein einheitlicher, sondern setzt sich aus mehreren terrassenförmig übereinander angeordneten Rändern zusammen, so daß diese in ihrer Gesamtheit fast die Hälfte des Substanzverlustes einnehmen. Der übrig bleibende zentrale Teil des Substanzverlustes ist demzufolge sehr tief und zeigt sich von einer gelbgrünen schmierigen Masse belegt. In der Umgebung des Substanzverlustes wenig Rötung, jedoch ist die ganze Stelle in weiterem Umkreis ziemlich, an Stelle des Substanzverlustes selbst sehr schmerzhaft. - Ein etwas kleinerer Herd an der radialen Seite der Grundphalanx des rechten Zeigefingers, von einer braunen festhaftenden Borke bedeckt, ein ebensolcher an der Kuppe des Fingers. An der nlnaren Seite des linken Daumens eine längsovale, mit einer dicken, nicht ablösbaren Kruste bedeckte Stelle. (Alle Verletzungen rühren nach Angabe des Patienten von Schnitten her. S. darüber unten.) Die Haut beider Hände und der distalen Teile der Arme ist im ganzen braun verfärbt.

2. R. An der rechten Mitteltingerkuppe ein unter bohnengroßer, scharf konturierter Substanzverlust mit terrassenförmig abfallenden Rändern, bis im Zentrum nur ein etwa linsengroßer Subslanzverlust übrig bleibt. In der Umgebung keine Reaktionserscheinungen. Ein ebensolcher Herd an dem radialen Rand der rechten Hand, wobei anch hier auffält, daß der zentrale Teil besonders tief ist. - Die Farbe beider Substanzverluste fast schwarz, die Haut an der rechten und linken Hand überhaupt stark braun. - An der linken Hand findet sich ebenfalls ein größerer Substanzverlust vom beschriebenen Aussehen dorsal am Interphalangealgelenk II/III des Zeigefingers, außerdem noch mehrere kleinere meist lineare von Borken bedeckte an den andern Fingern beider Hände. Alle erkrankten Partien sind hochgradig schmerzhaft und zwar besonders auf Berührung empfindlich, jedoch hat Patient auch spontan heftige Schmerzen.

3. M. Am Inter digitalgelenk II/III des rechten 4. Fingers und zwar an der radialen Seite ein etwa bobnengroßer, scharf konturierter Substanzverlust, der von einem doppelten Rande umgeben ist. Der erste äußere Rand bildet genau ein Oval und ist eben noch deutlich bemerkbar, da der durch ihn begrenzte Teil des Substanzverlustes nur wenig unter dem Niveau der Haut liegt und daher nur leicht deprimiert erscheint. Etwa 1-2 $m m$ einwärts von diesem Rande ist ein $\mathrm{z}$ weiter ebenfalls scharf ausgeschitten, jedoch in seiner Form mehr unregelmäBig, da er nur im allgemeinen der Ellipsenform folgt, an einigen Stellen aber Zacken 
aufweist, die jedoch ebenfalls scharf ausgeschnitten erscheinen. Dieser Rand ist steil und sehr tief, so daß der von ihm begrenzte zentrale Anteil des Substanzverlustes bedeutend unter dem Niveau der Haut liegt. Besonders ist dies bei der distalen Partie zu sehen, während der proximale Anteil weniger tief liegt. Ein schmutzig gelbgrünlicher Belag bedeckt den Grund -des Snbstanzverlustes, der wieder sebr schmerzhaft ist. Die Haut an den Phalangen II und III erscheint leicht gerötet.

An dem Interphalangealgelenk $\mathrm{I} / \mathrm{II}$ des linken Mittelfingers an der Ulnarseite findet sich ein vollständig gleich aussehender, nur etwas seichterer und mehr längsovaler Substanzverlust, welcher ebenfalls die beiden Ränder deutlich zeigt. Um ihn herum sind die Reaktionserscheinungen, Rötung und Schmerzhaftigkeit mehr ausgesprochen. Außer diesen beiden zunächst in die Augen fallenden Substanzverlusten noch mehrere andere meist mit Krusten bedeckte kleinere, zum Teil ungefähr oval, zum Teil mehr linear. Die Haut beider Hände gelbbraun verfärbt, die Krusten dunkler, fast brannschwarz.

Vergleichen wir also die Befunde, wie sie sich bei diesen 3 Patienten ergeben, miteinander, so sehen wir auch hier die Bildung scharfkonturierter Substanzverluste, die vor allem durch ihre Tiefe im Zentrum auffallen, während die Randpartien weniger tief erscheinen. Wenigstens ist uns dies bei den erwähnten Fällen aufgefallen, woraus natürlich nicbt ein Schluß als Verallgemeinerung gezogen werden darf. Es möge dieser Umstand daher nur erwähnt werden, da er besonders auffiel, wenn wir diese Verätzungen mit den Kalkverätzungen verglichen. Denkt man sich eine Ebene senkrecht auf die Ulzeration errichtet, so fällt die jeweilige Schnittlinie der beiden Flächen verschieden aus. Bei den Kalkverätzungen ergibt sich ungefähr ein Halbkreis, während bei den Schwefelsäureverätzungen zwei im allgemeinen einen Winkel miteinander bildende Zickzacklinien sich ergeben und nur am Grunde erst eine diese Linien verbindende Kurve entsteht. Vielleicht liegt der Grund der Verschiedenheit in der Konfiguration der substanzverluste darin, daß bei den Kalkverätzungen die ätzende Substanz immer nur auf das direkt angrenzende Gebiet einwirkt und so gewissermaßen nur eine Grube gräbt, die so groß ist, daß das Ätzmaterial darin Platz finden kann, während bei den Schwefelsäureverätzungen die Wirkung der Flïssigkeit besonders intensiv und tiefgreifend im Zentrum ist, jedoch auch noch ziemlich weit gegen die Peripherie hin besteht, da ja diese einer Flüssigkeit leichter 
zugänglich ist als einer relativ festen Substanz, wie es der gelöschte Kalk ist.

Daß auch in den letzterwähnten Fällen die Verletzung der Haut eine wesentliche Rolle spielt, geht aus den Angaben hervor, welche die Arbeiter über ihre Beschäftigung gemacht haben und die, was nebenbei erwähnt werden möge, alle drei in einem Betriebe arbeiten. Die Schwefelsäure wird dort verwendet, damit von Eisenblechen der Rost entfernt werde, zu welchem Zwecke eine schwefelsäurehältige Beize hergestellt wird. Nach der Behandlung mit dieser Beize werden die Bleche mit Zangen herausgenommen und mit reinem Wasser abgewaschen, worauf sie dann der weiteren Bearbeitung (dem Verzinken) zugeführt werden können. Selbstrerständlich gebrauchen die Arbeiter beim Anfassen der Bleche nicht nur die Zangen sondern auch direkt ibre Hände, so daß sie durch die scharfkantigen Ränder leicht Schnittwunden akquirieren. An diesen Stellen kommt es dann auch zum Auftreten der Ulzerationen, die sich durch ihre Schmerzhaftigkeit bald hindernd entgegenstellen, während sie anfangs - wenigstens nach Angabe unserer drei Patienten - nicht beobachtet werden. An den intakten Stellen ruft die Beize nur eine mehr oder minder intensive Braunfärbung der Haut hervor, von der die Patienten natürlich nicht belästigt. werden, während die Schmerzhaftigkeit der Ulzerationen jene früher gelegentlich eines Falles von Kalkverätzung beschriebene Ängstlichkeit und Unbeholfenheit im Gebrauch der Hände hervorruft, welche uns auch ein objektives Zeichen der Schwere des Prozesses gibt, abgesehen von den rein lokalen Veränderungen.

Vom Standpunkt der Arbeitsfähigkeit aus betrachtet, mußten auch diese 3 Patienten in den Krankenstand aufgenommen werden, dessen Dauer aber hier eine bedeutend längere gewesen ist, da sie bei den ersten beiden Patienten einen Zeitraum von einem Monat betrug und der dritte derzeit schon 10 Tage im Krankenstand ist und erst etwas gebessert erscheint. Somit sind auch diese Patienten einer schweren Schädlichkeit ausgesetzt. In unseren Fällen waren es Arbeiter in einer Verzinkerei, jedoch sind dieselben Schwefelsäureverätzungen auch bei den Arbeitern in Hutfabriken, wie Sachs (6) betont, zu 
sehen, wo ebenfalls Schwefelsäure in ziemlich konzentrierter Form verwendet wird. Wenn daber durch die Anführung einer Reihe solcher Fälle gezeigt worden ist, daß es sich hier nicht um einzeln stehende Fälle, sondern um solche handelt, die in bestimmten Betrieben vorkommen und durch die Art derselben bedingt sind, so muß dies auch zu der Erkenntnis führen, daß es sich nicht um relativ gleichgültige, weil zufällige Krankheiten handelt, sondern daß diese mit den notwendigen Hantierungen der Arbeiter im Zusammenhang stehen, und daß diese daher eines Schutzes gegen das Entstehen solcher gewerblicher Erkrankungen bedürfen.

\section{Literatur.}

1. Wiener klinische Wochenschrift 1903.

2. Gazette des Hopitaux 1. Februar 1906.

2a. Annales de Dermatologie et de Syphiligraphie 1901.

3. Th. de Paris 1900/1901.

4. Handbuch der Arbeiterkrankheiten von Weyl 1908.

5. Layet Gewerbepathologie und Gewerbehygiene.

6. Protokolle der Sitzungen der Wiener dermatologischen Gesellschaft 1908. 
Die Erklärung der Abbildungen auf Taf. $I$ ist dem Texte zu entnehmen. 

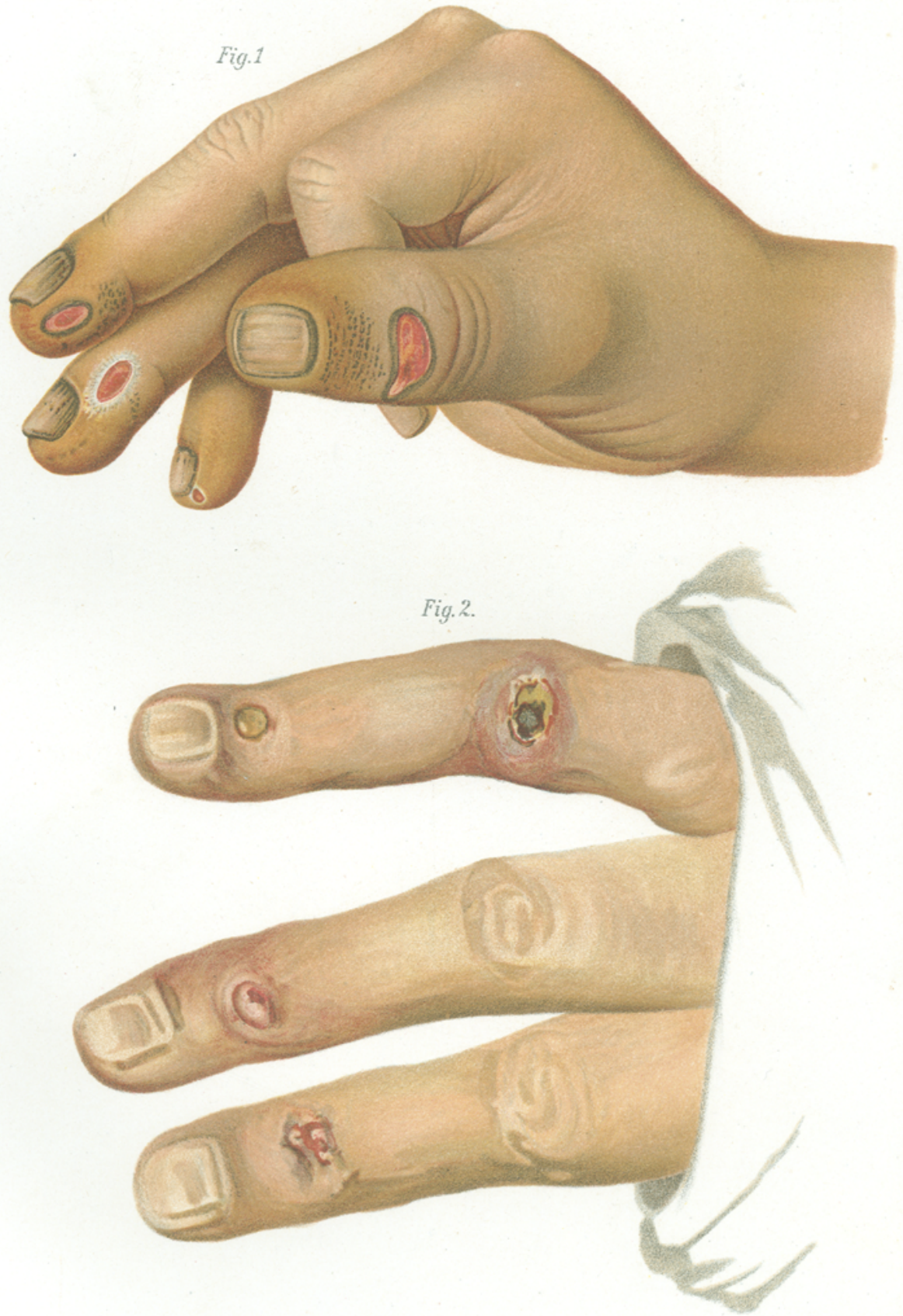

Neugebauer: Verätzungen an den Händen der. Metallarbeiter. 